TITLE:

\title{
Suppression and control of prethermalization in multicomponent Fermi gases following a quantum quench
}

\section{$\operatorname{AUTHOR}(\mathrm{S})$ :}

Chen-How-Huang; Takasu, Yosuke; Takahashi, Yoshiro; Cazalilla, Miguel A.

\section{CITATION:}

Chen-How-Huang ...[et al]. Suppression and control of prethermalization in multicomponent Fermi gases following a quantum quench. Physical Review A 2020, 101(5): 053620.

\section{ISSUE DATE:}

2020-05

URL:

http://hdl.handle.net/2433/252476

RIGHT:

(C2020 American Physical Society; 許諾条件に基づいて掲載しています $\circ$ 


\title{
Suppression and control of prethermalization in multicomponent Fermi gases following a quantum quench
}

\author{
Chen-How Huang, ${ }^{1}$ Yosuke Takasu $\odot,{ }^{2}$ Yoshiro Takahashi, ${ }^{2}$ and Miguel A. Cazalilla ${ }^{1,3,4}$ \\ ${ }^{1}$ Department of Physics, National Tsing Hua University, Hsinchu 30013, Taiwan \\ ${ }^{2}$ Department of Physics, Graduate School of Science, Kyoto University, Kyoto 606-8502, Japan \\ ${ }^{3}$ National Center for Theoretical Sciences (NCTS), Hsinchu City, Taiwan \\ ${ }^{4}$ Donostia International Physics Center (DIPC), Manuel de Lardizabal, 4 San Sebastian, Spain
}

(Received 29 October 2019; revised manuscript received 27 March 2020; accepted 6 April 2020; published 12 May 2020)

\begin{abstract}
We investigate the mechanisms of control and suppression of prethermalization in $N$-component alkalineearth-metal gases. To this end, we compute the short-time dynamics of the instantaneous momentum distribution and the relative population for different initial conditions after an interaction quench, accounting for the effect of initial interactions. We find that switching on an interaction that breaks the $\mathrm{SU}(N)$ symmetry of the initial Hamiltonian, thus allowing for the occurrence of spin-changing collisions, does not necessarily lead to a suppression of prethermalization. However, the suppression will be most effective in the presence of SU(N)-breaking interactions provided the number of components $N \geqslant 4$ and the initial state contains a population imbalance that breaks the $\mathrm{SU}(N)$ symmetry. We also find the conditions on the imbalance initial state that allow for a prethermal state to be stabilized for a certain time. Our study highlights the important role played by the initial state in the prethermalization dynamics of multicomponent Fermi gases. It also demonstrates that alkaline-earth-metal Fermi gases provide an interesting playground for the study and control of prethermalization.
\end{abstract}

DOI: 10.1103/PhysRevA.101.053620

\section{INTRODUCTION}

Dilute Fermi gases of akaline-earth-metal atoms (AEMAs) like ${ }^{173} \mathrm{Yb}$ or ${ }^{87} \mathrm{Sr}$ exhibit a remarkable unitary symmetry at ultracold temperatures [1-3]. Such symmetry is ultimately a consequence of their closed-shell (ground-state) electronic structure, ${ }^{1} S_{0}$ : For AEMAs, the atomic total angular momentum $\boldsymbol{F}$ in their ground state equals the nuclear spin $\boldsymbol{I}$. Due to the weakness of hyperfine interactions, $\boldsymbol{I}$ is essentially decoupled from the electronic degrees of freedom. Thus ultracold gases of AEMAs can be viewed as spin- $I$ particles interacting with a pseudopotential that is independent of their nuclear spin orientation, $I_{z}$, and therefore invariant under the larger unitary group $\mathrm{SU}(N=2 I+1)$. The accuracy of this $\mathrm{SU}(N)$-symmetric description of interaction has been confirmed experimentally $[4,5]$

Recently, this property has attracted a great deal of interest in connection with the possibility of quantum emulation of SU(N)-symmetric models of interest to condensed matter physicists [1,2,6-19]. In recent years, many experiments along this direction have been carried out [20-26] (see also [3] for a recent review). In addition, the realization of $\mathrm{SU}(N>2)$ symmetric many-body systems is relevant to the understanding of some aspects of the strong force that binds quarks into nucleons $[27,28]$. Indeed, as a quantum emulator of an $\mathrm{SU}(N)$ interacting fermion gas for which $N$ can be as large as 10 , ultracold gases of AEMAs can provide an ultracold realization of certain toy models of quantum chromodynamics (QCD) [28]. In this regard, it is interesting to explore any further connections between ultracold gases of AEMAs and quarkgluon physics. Indeed, an idea that has recently emerged in the study of the quark-gluon plasma is the existence of prethermalized states [29]. These states are characterized by the rapid establishment of a kinetic temperature while, at the same time, the distribution of the eigenmodes of the system has not reached thermal equilibrium as described by the Fermi-Dirac or Bose-Einstein distributions. Prethermalized states have been extensively discussed in relation to the nonequilibrium dynamics of ultracold atomic gases [30-49]. In earlier work, it was also shown [32] that prethermalization can be also linked to the existence of an integrable truncated version of the Hamiltonian that describes the short-time dynamics [32] and can be related [32] to the nonthermal states occurring in integrable systems, which are described by the generalized Gibbs ensemble [42,45,46,50-58]. However, for nonintegrable systems, the system will eventually relax at long times to a thermal state described by a standard Gibbs ensemble [59-68]. If the breaking of integrability is weak, the prethermalized state emerging at short times can be fairly long lived [30,31,34,34-41,44,45,47,48,69-78].

Understanding the conditions under which a system exhibits prethermalization is an important ongoing research effort. In this work, we investigate the control and suppression of the prethermalized behavior by the choice of the initial state and the symmetries of the post-quench Hamiltonian. Mathematically, the emergent $\mathrm{SU}(N=2 I+1)$ symmetry described above forbids spin-changing collisions that change the relative population of the different spin components (cf. Fig. 1). Thus, we shall consider the short-time dynamics of an AEMA Fermi gas following a sudden interaction quench in which the postquench Hamiltonian breaks the $S U(N)$ symmetry. We analyze under which conditions this system exhibits prethermalization 


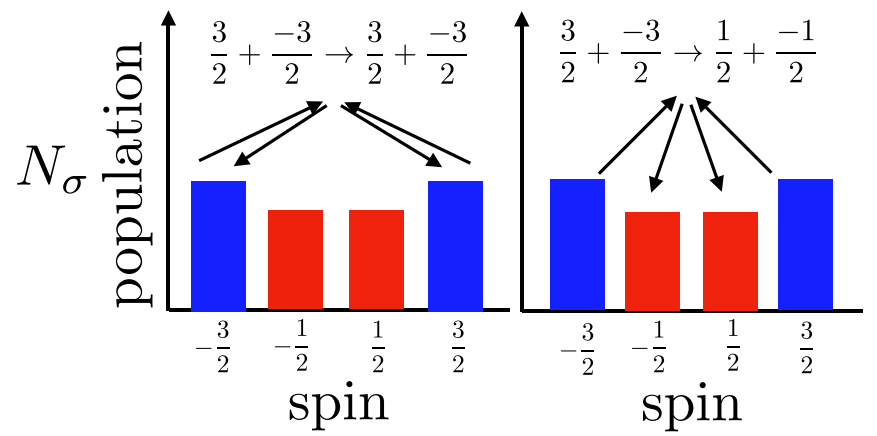

FIG. 1. Two possible scattering processes in an SU(4) Fermi gas following a quench of the interaction that breaks the emergent SU(4) symmetry, starting from an initial state with population imbalance. Right panel: Spin-changing collision (as understood in this work) defines a two-atom collision that modifies the relative spin populations. Left panel: Other scattering processes that conserve total spin and do not change the relative spin-population.

and, in particular, how the emergence of prethermalization is affected by allowing for spin-changing collisions. It is known [30-32,39] that the existence of prethermalized states in Fermi gases is related to the lack of phase space for collisions that increase the number of particle-hole pairs. Thus, thermalization is only possible after enough phase space has been created for such collisions to efficiently take place $[30,31,39]$. Thermalization happens more efficiently as the strength of the quenched interaction is increased, as has been confirmed numerically $[69,76]$. In this work we further analyze this issue and show that the mere existence of phase space in the initial state allowing for collisions that increase the number of particle-hole pairs is a necessary but not sufficient condition for the suppression of prethermalization. Furthermore, the existence of matrix elements of the interaction allowing for such collisions is not sufficient if it is not accompanied by the existence of phase space in the initial state for the collisions to take place. Nevertheless, as we show below, it is possible to find certain types of initial states for which the rate at which such collisions happen at intermediate times vanishes.

The rest of this article is organized as follows. The details of the model as well as the methods employed for the study of prethermalization are discussed in Sec. II. In Sec. III, the details of the calculations of the instantaneous momentum distribution are provided. In Sec. IV, we describe the quench dynamics in the two-component gas. In Sec. V we report the results for the four-component gas and discuss the effects of spin-changing collisions and the spin-imbalance in the initial state. In Sec. VII, we provide the conclusions of this work and summarize the main results. Some details of the calculations are given in Appendix A. Henceforth, we shall work in units where $\hbar=1$.

\section{MODEL AND METHODS}

An interaction quench in an AEMA ultracold gas can be described by means of the following Hamiltonian:

$$
H(t)=H_{0}+U_{1}(t)
$$

$$
\begin{gathered}
H_{0}=K+U_{0}, \\
K=\sum_{\boldsymbol{p} \sigma} \epsilon_{p} c_{\boldsymbol{p} \sigma}^{\dagger} c_{\boldsymbol{p} \sigma},
\end{gathered}
$$

where $\epsilon_{p}=p^{2} / 2 m$ is the single-particle dispersion, and $c_{\boldsymbol{p} \sigma}, c_{\boldsymbol{p} \sigma}^{\dagger}$ are the annihilation and creation operators of fermions with momentum $p$ and spin $\sigma$ obeying $\left\{c_{\boldsymbol{p} \alpha}, c_{\boldsymbol{k} \beta}^{\dagger}\right\}=$ $\delta_{\alpha, \beta} \delta_{p, \boldsymbol{k}}$ (and anticommuting otherwise); $U_{0}$ is the initial $\left(\mathrm{SU}(N)\right.$-symmetric [1-3]) interaction and $U_{1}(t)=\theta(t) U_{1}$ is the quenched interaction (see below), $\theta(t)$ being Heaviside's step function, which describes a sudden quench of the interaction term $U_{1}$. The generalization of our methods to other types of quenches has been presented in Ref. [79], where special attention was paid to the proper definition of the energy and the asymptotic behavior of the momentum distribution out of equilibrium. In the sudden quench limit, the calculation of the dynamics of the total energy is less involved; see the discussion at the end of this section.

In general, for ultracold Fermi gases of spin- $F$ atoms, the generalization of Lee-Huang-Yang pseudopotential that describes the two-particle collisions in the $s$-wave channel reads [80]

$$
\begin{aligned}
U_{1}= & \sum_{J=0,2, \ldots}^{2 F-1} \frac{g_{J}}{2 V} \sum_{M=-J}^{J} \sum_{\alpha \beta \gamma \delta}\langle F F \alpha \beta \mid J M\rangle\langle J M \mid F F \gamma \delta\rangle \\
& \times \sum_{p k q \boldsymbol{r}} c_{\boldsymbol{p} \alpha}^{\dagger} c_{\boldsymbol{k} \beta}^{\dagger} c_{\boldsymbol{q} \gamma} c_{\boldsymbol{r} \delta} \delta_{p+k, q+r},
\end{aligned}
$$

where $\langle\alpha \beta \mid F F m J\rangle$ are Clebsch-Gordan coefficients. The couplings that parametrize the short-range interaction are $g_{J}=$ $8 \pi a_{s}^{J} / m$, where $a_{s}^{J}$ are the $s$-wave scattering lengths of the scattering channel with total spin $J, m$ is the atom mass, and $V$ is the volume of the system. However, before the interaction quench, the AEMAs are in their ground state and all the scattering lengths $a_{s}^{J}$ are identical with very high accuracy, which results in the emergent $\mathrm{SU}(N)$ symmetry [1-3] mentioned above. Thus, the initial interaction reads

$$
U_{0}=\frac{g_{i}}{2 V} \sum_{p k q \boldsymbol{r}} c_{\boldsymbol{p} \alpha}^{\dagger} c_{\boldsymbol{k} \beta}^{\dagger} c_{\boldsymbol{q} \beta} c_{\boldsymbol{r} \alpha} \delta_{\boldsymbol{p}+\boldsymbol{k}, \boldsymbol{q}+\boldsymbol{r}},
$$

where the coupling $g_{i}=8 \pi a_{s} / m, a_{s}$ being the scattering length. However, suddenly turning on $U_{1}$ which contains different values of $g_{J}$ (i.e., $g_{0} \neq g_{2} \neq \cdots$ ) breaks the $\operatorname{SU}(N)$ symmetry while respecting spin rotation symmetry. This means that in the two-particle scattering events the total (hyperfine) spin of the colliding particles is still conserved but the spins of the colliding particles can change. In that case, we speak of spin changing collisions (SCCs; see Fig. 1, right panel).

Unfortunately, for gases of AEMAs the kind of interaction quench envisaged above that breaks the emergent $\mathrm{SU}(N)$ symmetry cannot be realized using magnetic Feshbach resonances because they are not accessible in the ground state due to their closed-shell electronic structure. However, by means of the so-called optical Feshbach resonances (OFR) [4,81-86] it is possible to (suddenly) enhance the values of the scattering lengths $a_{s}^{J}$. To this end, a laser is used to couple a pair of 
colliding atoms with an excited bound state, which induces a Feshbach resonance and modifies the scattering lengths $a_{s}^{J}$. This method for enhancing the interaction violates the emergent $\mathrm{SU}(N)$ symmetry of the resulting interaction since the ground state is coupled to an excited state that possesses a hyperfine structure. As a result, it should possible to observe the dynamics of the initially $\mathrm{SU}(N)$-symmetric gas that is subject to an $\mathrm{SU}(N)$-symmetry-breaking interaction quench. The price to pay for the use of OFR is the introduction of inelastic losses, which result from the real excitation to the excited bound state of a pair of AEMAs. Inelastic losses will provide an additional mechanism for the suppression of prethermalization. Nevertheless, as shown below (see, e.g., Figs. 4 and 8) the characteristic timescale for the emergence of prethermalization is determined by the inverse of the (average) Fermi energy in the initial state. In order to assess the effect of inelastic collisions, we can evaluate the ratio of the rate of the accompanying loss in the OFR to the Fermi energy of the gas from Eqs. (6)-(9) in Ref. [82]. Thus, we find that the ratio can be much smaller than 1 for values of $a_{s}^{J} / a_{s}$ as large as $10^{4}$, where $a_{s}^{J}$ is the largest scattering length in the quenched interaction [cf. Eq. (4)]. Let us recall that, according to our perturbative analysis (see below), the strength of the interaction controls the magnitude of the effects (as long as prethermalization is accessible using perturbation theory; see the discussion below Eq. (7) and also [69,76]). Thus, it is safe to envisage values of $a_{s}^{J} / a_{s} \gtrsim 10$, which can lead to observable effects while keeping the effect of the inelastic losses at a negligible level.

Following previous work [29-32,39,45,46,76,77,87,88], we shall study the evolution of the instantaneous momentum distribution and the total energy in order to identify the prethermalized regime; i.e., we compute

$$
\begin{gathered}
n_{p \sigma}(t)=\left\langle\Psi(t)\left|c_{p \sigma}^{\dagger} c_{p \sigma}\right| \Psi(t)\right\rangle, \\
E_{\mathrm{tot}}(t)=\langle\Psi(t)|H(t)| \Psi(t)\rangle,
\end{gathered}
$$

where $|\Psi(t)\rangle$ is the solution of the time-dependent Schrödinger equation for the Hamiltonian $H(t)$ [cf. Eq. (2)]. In the following section, we compute the short-time dynamics of $n_{p \sigma}(t)$ using perturbation theory to the lowest nontrivial orders in the initial and quench interactions. The second-order results obtained below are valid for times that fulfill the condition $[30,31] \epsilon_{F} t \lesssim\left(g_{\max }^{J} k_{F}^{3} / \epsilon_{F}\right)^{-3} \simeq\left(k_{F} a_{\max }^{J}\right)^{-3}$, where $g_{\max }^{J}\left(a_{\max }^{J}\right)$ is the largest coupling (scattering length) in the quenched interaction and $\epsilon_{F}\left(k_{F}\right)$ is the mean Fermi energy (momentum).

From the instantaneous momentum distribution, we obtain the total particle number of each spin component,

$$
N_{\sigma}(t)=\sum_{p} n_{p \sigma}(t),
$$

as well the discontinuity of the momentum distribution at Fermi momentum:

$$
Z_{\sigma}(t)=\lim _{\delta \rightarrow 0^{+}}\left[n_{p_{F \sigma}+\delta, \sigma}(t)-n_{p_{F \sigma}-\delta, \sigma}(t)\right] .
$$

Below, when discussing prethermalization, we will focus on $Z_{\sigma}(t)$ rather than on the full momentum distribution.
For a sudden quench, the dynamics of total energy can be obtained by resorting to energy conservation. Consider the evolution of the total energy for times $t>0$ : The state of the system is described by $|\Psi(t)\rangle=e^{-i\left(H_{0}+U_{1}\right) t}|\Psi(0)\rangle$. Hence,

$$
\begin{aligned}
E_{\mathrm{tot}}(t>0) & =\langle\Psi(t)|H(t)| \Psi(t)\rangle \\
& =\left\langle\Psi(0)\left|\left(H_{0}+U_{1}\right)\right| \Psi(0)\right\rangle \\
& =E_{0}+\left\langle\Psi(0)\left|U_{1}\right| \Psi(0)\right\rangle,
\end{aligned}
$$

where $E_{0}=\left\langle\Psi(0)\left|H_{0}\right| \Psi(0)\right\rangle$. In other words, the total energy is a constant for $t>0$, and for any time $t$ it exhibits rather simple evolution dynamics:

$$
E_{\text {tot }}(t)=E_{0}+\theta(t)\left\langle\Psi(0)\left|U_{1}\right| \Psi(0)\right\rangle .
$$

The above result implies that the total energy immediately reaches its final (thermal) value after the quench. For a Dirac-delta interaction (also called single-channel model), it is not possible to mathematically define the instantaneous kinetic energy. This is because the instantaneous momentum distribution $n_{k \sigma}(t)$ behaves as $k^{-4}$ for $k \gg k_{F}$ [79], which renders the integral $E_{\mathrm{kin}}=\sum_{k, \sigma} \epsilon_{k} n_{k \sigma}(t)$ divergent. Thus, we shall define the prethermalized regime as a state in which the total energy has reached its (final) thermal value while the momentum distribution has not. This means that the existence of a prethermalized regime can entirely be inferred from the existence, for a certain time following the quench, of a quasistationary, nonthermal momentum distribution. In Fermi systems, this is manifested by a plateau in the evolution of the instantaneous discontinuity at the Fermi momentum, $Z_{\sigma}(t)$ [cf. Eq. (9)].

\section{INSTANTANEOUS MOMENTUM DISTRIBUTION}

In this section, we describe how the time evolution of the instantaneous momentum distribution is obtained. Assuming that the interaction strength is weak, we shall compute the evolution of a given observable $O$ in a perturbative series in the total interaction, $V(t)=e^{i K t}\left[U_{0}(t)+U_{1}(t)\right] e^{-i K t}$, where we used the fact that the initial interaction $U_{0}(t)=U_{0} e^{-\eta|t|}$ is adiabatically switched on (off) at a rate $\eta \rightarrow 0^{+}$. Thus,

$$
\begin{aligned}
O(t)= & \frac{\left\langle\mathrm{GS}, v\left|\mathcal{T}\left[e^{-i \int_{C} d t V(t)} O(t)\right]\right| \mathrm{GS}, v\right\rangle}{\left\langle\mathrm{GS}, \nu\left|\mathcal{T}\left[e^{-i \int_{C} d t V(t)}\right]\right| \mathrm{GS}, v\right\rangle} \\
= & \langle O\rangle-i \int_{C} d t_{1}\left\langle\mathcal{T}\left[V\left(t_{1}\right) O(t)\right]\right\rangle \\
& +\frac{(-i)^{2}}{2 !} \int_{C} d t_{1} d t_{2}\left\langle\mathcal{T}\left[V\left(t_{1}\right) V\left(t_{2}\right) O(t)\right]\right\rangle_{c}+\cdots,
\end{aligned}
$$

where $\langle O\rangle=\langle\mathrm{GS}, v|O| \mathrm{GS}, v\rangle$. The times $t_{1}, t_{2}, \ldots$ all lie on the closed contour $C$ shown in Fig. 2 and $\mathcal{T}$ is the timeordering symbol on $C$. The state $|\mathrm{GS}, v\rangle$ denotes a noninteracting state, which is characterized by a particular ratio $v$ of the population of the different spin components (the definition of $v$ depends on the number of components; see below). Strictly speaking, the denominator of Eq. (14) equals unity, but it is needed when expanding in powers of $V(t)$ in order 


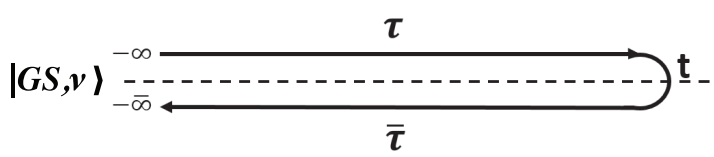

FIG. 2. Closed-time contour $C$. Times $\tau$ and $\bar{\tau}$ lie on the timeordered and anti-time-ordered branches, respectively. $\tau$ is earlier than $\bar{\tau}$ in contour ordering. The turning point $t$ is the time of at which observable in which we are interested is evaluated. $|\mathrm{GS}, v\rangle$ is the initial state, which we take to be the ground state of a noninteracting $N$-component Fermi gas characterized by a certain population ratio $v$ (see the discussion below).

to cancel disconnected terms resulting from the application of Wick's theorem to the above expression.

Previous work [30-32,39,45,46,76,77,87,88] has established that prethermalization is accessible through perturbation theory. In the problem of interest here, the perturbation series for the observable $O$ can be organized as a double perturbative expansion in powers of $U_{0}$ and $U_{1}$, i.e.,

$$
\begin{aligned}
\langle O(t)\rangle= & O^{(0,0)}+O^{(0,1)}(t)+O^{(1,0)} \\
& +O^{(1,1)}+O^{(0,2)}(t)+O^{(2,0)}(t)+\cdots,
\end{aligned}
$$

where $O^{(n, m)}(t)$ denotes the term that is $n$th order in the initial interaction $U_{0}$ and $m$ th order in the quenched interaction $U_{1}$. Next we set $O=c_{\boldsymbol{p} \sigma}^{\dagger} c_{\boldsymbol{p} \sigma}$, and thus,

$$
\begin{aligned}
n_{p \sigma}(t)= & n_{p \sigma}^{(0,0)}(t)+n_{p \sigma}^{(1,0)}(t)+n_{p \sigma}^{(0,1)}(t) \\
& +n_{p \sigma}^{(1,1)}(t)+n_{p \sigma}^{(2,0)}(t)+n_{p \sigma}^{(0,2)}(t)+\cdots,
\end{aligned}
$$

where $n_{p \sigma}^{(0,0)}(t)=n_{p \sigma}^{0}$. In the closed time contour $C$, we can express the other $n_{p \sigma}^{(n, m)}(t)$ in terms of the self-energy and propagator matrices which, to second order in the total interaction, read

$$
\begin{aligned}
& n_{p \sigma}^{(1,0)}(t)=\int_{C} d t_{1} \mathcal{G}_{p \sigma}\left(t, t_{1}\right) \Sigma_{p \sigma}^{(0,1)}\left(t_{1}\right) \mathcal{G}_{p \sigma}\left(t_{1}, t\right), \\
& n_{p \sigma}^{(0,2)}(t)=\int_{C} d t_{1} \mathcal{G}_{p \sigma}\left(t, t_{1}\right) \Sigma_{p \sigma}^{(0,1)}\left(t_{1}\right) \mathcal{G}_{p \sigma}\left(t_{1}, t,\right) \\
& n_{p \sigma}^{(2,0)}(t)=\int_{C} d t_{1} d t_{2} \mathcal{G}_{p \sigma}\left(t, t_{2}\right) \Sigma_{p \sigma}^{(2,0)}\left(t_{2}, t_{1}\right) \mathcal{G}_{p \sigma}\left(t_{1}, t\right), \\
& n_{p \sigma}^{(1,1)}(t)=\int_{C} d t_{1} d t_{2} \mathcal{G}_{p \sigma}\left(t, t_{2}\right) \Sigma_{p \sigma}^{(1,1)}\left(t_{2}, t_{1}\right) \mathcal{G}_{p \sigma}\left(t_{1}, t\right) .
\end{aligned}
$$

The propagator $\mathcal{G}_{p \sigma}(a, b)$ is defined in Eq. (A2), and $\Sigma_{p \sigma}^{(1)}\left(t_{1}\right)$, $\Sigma_{p \sigma}^{(2)}\left(t_{2}, t_{1}\right)$ can be computed using Feynman diagrams (see Appendix A).

For the calculation of equal-time expectation values, we choose the time argument of the observable $(t)$ to lie slightly before the turning point of the contour $C$, which is on the time ordered $(\tau)$ branch. In this case, the fermion propagators must be obtained from Eq. (A1), which yields

$$
\mathcal{G}_{p \sigma}(t, b)=e^{-i \epsilon_{p}(t-b)}\left(\begin{array}{cc}
1-n_{p \sigma}^{0} & -n_{p \sigma}^{0} \\
0 & 0
\end{array}\right),
$$

where $\epsilon_{p}=p^{2} /(2 m)$ is the single-particle kinetic energy. The nonvanishing entries correspond to $b$ lying either before or after $t$ on the contour $C$. Similarly,

$$
\mathcal{G}_{p \sigma}(a, t)=e^{-i \epsilon_{p}(a-t)}\left(\begin{array}{cc}
-n_{p \sigma}^{0} & 0 \\
1-n_{p \sigma}^{0} & 0
\end{array}\right),
$$

and the two nonzero entries correspond to $a$ lying before or after $t$ on the contour $C$. Combining Eq. (18), the propagators [Eqs. (19) and (20)] and the second-order corrections to the self-energy [Eqs. (A17) to (A20)], we arrive at

$$
\begin{aligned}
n_{\boldsymbol{p} \sigma}^{(2)}(t)= & -\frac{2}{V^{2}} \sum_{\alpha \beta \gamma} \sum_{\boldsymbol{p k q r}} Q_{p \boldsymbol{p} \boldsymbol{r} \boldsymbol{r}}^{\sigma \alpha \beta \gamma} \int_{-\infty}^{t} d t_{1} \int_{-\infty}^{t} d t_{2} e^{i E_{p k q r}\left(t_{1}-t_{2}\right)} \\
& \times\left[g_{f}^{(2)}(\sigma, \alpha ; \beta, \gamma) \theta\left(t_{1}\right) \theta\left(t_{2}\right)+g_{i}^{2} \delta_{\sigma, \beta} \delta_{\alpha, \gamma} e^{-\eta\left(\left|t_{1}\right|+\left|t_{2}\right|\right)}+2 g_{i} g_{f}^{(1)}(\sigma, \alpha ; \sigma, \alpha) \delta_{\sigma \beta} \delta_{\alpha \gamma} \theta\left(t_{1}\right) e^{-\eta\left|t_{2}\right|}\right], \\
= & -\frac{2}{V^{2}} \sum_{\boldsymbol{k} \boldsymbol{q} \boldsymbol{r}} \sum_{\alpha \beta \gamma} Q_{p k \boldsymbol{k} \boldsymbol{r}}^{\sigma \alpha \beta}\left\{\frac{g_{i}^{2} \delta_{\sigma \beta} \delta_{\alpha \gamma}}{E_{p k q r}^{2}}+\left[g_{f}^{(2)}(\sigma, \alpha ; \beta, \gamma)+g_{i} g_{f}^{(1)}(\sigma, \alpha ; \sigma, \alpha) \delta_{\sigma \beta} \delta_{\alpha \gamma}\right] F\left(E_{p k q r}, t\right)\right\}
\end{aligned}
$$

where $g_{f}^{(1)}$ and $g_{f}^{(2)}$ are given by the interaction strengths

$$
\begin{gathered}
g_{f}^{(1)}(\sigma, \alpha ; \sigma, \alpha)=\sum_{J=0,2, \ldots} \sum_{M} g_{J}\langle J M \mid F F \sigma \alpha\rangle\langle F F \sigma \alpha \mid J M\rangle, \\
g_{f}^{(2)}(\sigma, \alpha ; \beta, \gamma)=\sum_{J_{1}, J_{2}=0,2, \ldots} \sum_{M_{1} M_{2}} g_{J_{1}} g_{J_{2}}\left\langle J_{1} M_{1} \mid F F \sigma \alpha\right\rangle\left\langle F F \beta \gamma \mid J_{1} M_{1}\right\rangle\left\langle J_{2} M_{2} \mid F F \sigma \alpha\right\rangle\left\langle F F \beta \gamma \mid J_{2} M_{2}\right\rangle,
\end{gathered}
$$

and we have defined $E_{p k q r}=\epsilon_{p}+\epsilon_{k}-\epsilon_{q}-\epsilon_{r}$ and introduced $F(E, t)$ to denote the result of the integration over $t_{1}$ and $t_{2}$ :

$$
F(E, t)=\frac{4 \sin ^{2}(E t / 2)}{E^{2}} .
$$

In Eq. (21) $Q_{p k q r}^{\sigma \alpha \beta \gamma}$ corresponds to the following expression:

$$
\begin{aligned}
Q_{p k q r}^{\sigma \alpha \beta \gamma}= & \delta_{p+k, q+r}\left[n_{p \sigma}^{0} n_{k \alpha}^{0}\left(1-n_{q \beta}^{0}\right)\left(1-n_{r \gamma}^{0}\right)\right. \\
& \left.-\left(1-n_{p \sigma}^{0}\right)\left(1-n_{k \alpha}^{0}\right) n_{q \beta}^{0} n_{r \gamma}^{0}\right] .
\end{aligned}
$$




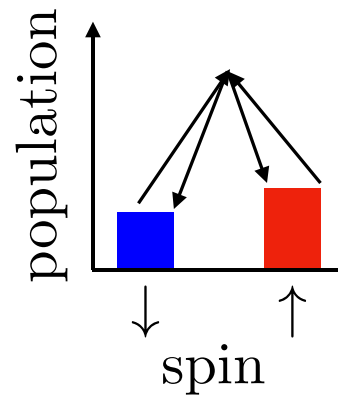

FIG. 3. Schematic representation of the only scattering process allowed in a two-component gas with Dirac-delta interactions. Notice that $(\uparrow+\downarrow) \rightarrow(\uparrow+\downarrow)$, thus preserving the spin populations for each spin component.

\section{TWO-COMPONENT FERMI GAS}

As a warmup, in this section we consider the twocomponent gas in order to show that breaking the $\operatorname{SU}(N=$ 2) symmetry of the initial state by introducing an initial population imbalance does not suppress prethermalization.

Let us first recall that, for a contact interaction in a two-component system, the pseudopotential in Eq. (4) is parametrized by a single coupling constant only, which is determined by the $s$-wave scattering length for atoms colliding with total $J=0, a_{s}$. Therefore, it is not possible to break the $\mathrm{SU}(N=2)$ symmetry of the interaction. Indeed, as shown in Fig. 3, the only scattering process in a two-component gas is of the type $(\uparrow+\downarrow) \rightarrow(\uparrow+\downarrow)$. This kind of scattering process preserves the populations of each spin component. Thus, even if the initial state contains a population imbalance (i.e., for $v=N_{\uparrow} / N_{\downarrow} \neq 1$ ), the time evolution following the quench cannot alter the ratio $v$. This conservation law alone protects the existence of the prethermalized regime.

In order to show that prethermalization is preserved, we have evaluated explicitly the time evolution of the discontinuity of the instantaneous momentum distribution at Fermi momenta, i.e., $Z_{\sigma}(t)$; see Fig. 4. Notice that, after a short transient, $Z_{\sigma}(t)$ displays a plateau for both spin components and the momentum distribution approaches a stationary behavior. This is a behavior characteristic of the prethermalized regime indicating that the momentum distribution is nonthermal, similarly to what was found in previous studies using different models and spin-unpolarized initial states [29,30,32,76]. The initial population imbalance is reflected in the prethermal value of $Z_{\uparrow}(t)$ being different from that of $Z_{\downarrow}(t)$. Indeed, we have checked that this result also applies to $N>2$ component Fermi gas. Thus, we conclude that, if the postquench Hamiltonian retains the $\mathrm{SU}(N)$ symmetry, the system shows prethermalization independently of the existence of population imbalance in the initial state (see discussion in the following section).

Next we consider the effects of the initial interaction. Although the interaction is $\operatorname{SU}(N=2)$ symmetric and therefore will not suppress prethermalization, for the sake of experimental interest it is worth analyzing its quantitative effect on the dynamics of the instantaneous momentum distribution. As a function of the ratio $a_{s} / a_{s}^{0}$, Fig. 5 shows the ratio of the prethermalized value of $Z_{\uparrow}(t)$ to its value in the ground state of $H_{0}+U_{1}=K+U_{0}+U_{1}$ (see Appendix A for the details of the calculation). Recall that the (initial) interaction strength is $g_{i}=8 \pi a_{s} / m$ and $a_{s}^{0}$ is the scattering length characterizing the quench interaction strength $g_{0}=8 \pi a_{s}^{0} / m$. Thus, the final interaction is equal to $8 \pi\left(a_{s}+a_{s}^{0}\right) / m$. The inset shows the full time dependence for a few values of the ratio $a_{s} / a_{s}^{0}$. The results shown in Fig. 5 can be summarized by the following relation:

$$
\frac{1-Z_{\sigma}^{s t}}{1-Z_{\sigma}^{e q}}=C\left(\frac{a_{s}}{a_{s}^{0}}\right),
$$

where $Z_{\sigma}^{s t}$ and $Z_{\sigma}^{e q}$ are the stationary and equilibrium values of the discontinuity at the Fermi momentum of the momentum distribution. The crossover function $C(x)$ takes the following limiting forms: $C(x \gg 1)=1$ and $C(x \ll 1)=2$, while interpolating in between for intermediate values of $x=a_{s} / a_{s}^{0}$. The $x \rightarrow 0$ limit, which corresponds to the noninteracting initial state, has been obtained in previous work [30-32]. The factor of 2 arises from the long-time limit of the sinus square in
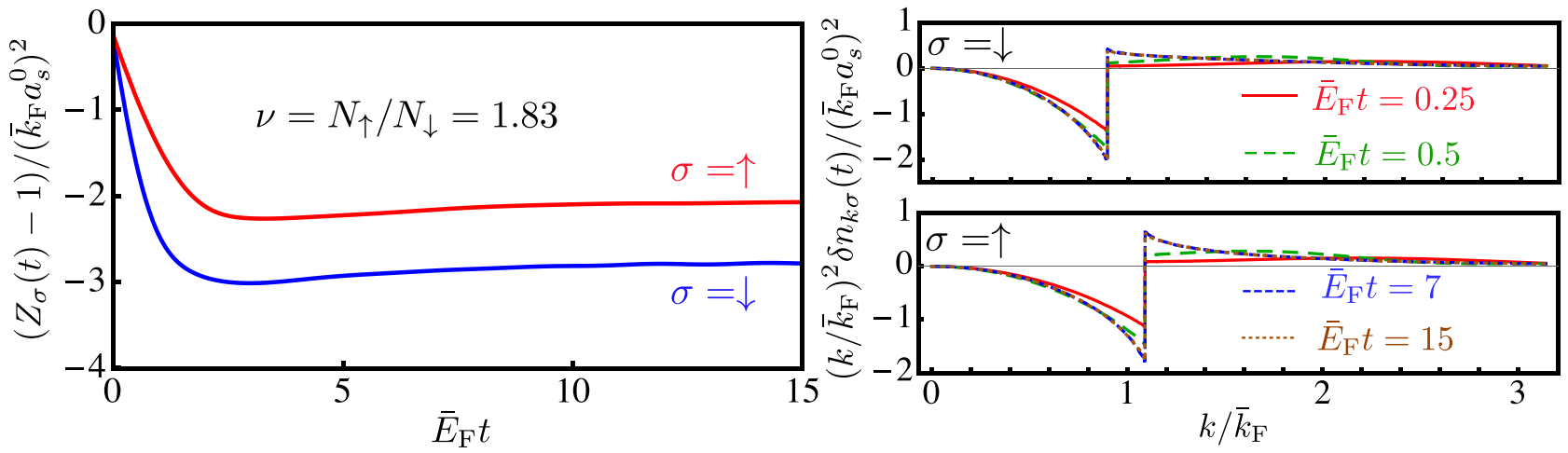

FIG. 4. Left panel: Dynamics of discontinuity of the momentum distribution at Fermi momentum $Z_{\sigma}(t)$ after a sudden interaction quench in a two-component Fermi gas. After a short transient, a plateau is observed indicating the existence of a prethermalized regime. Right panel: Dynamics of the change in momentum distribution $\left(k / \bar{k}_{F}\right)^{2} \delta n_{k \sigma}$ show stationary behaviors for both components. The $\left(k / \bar{k}_{F}\right)^{2}$ is chosen since $\sum_{k}(\cdots)=\int(\cdots) k^{2} d k d \Omega /(2 \pi)^{3}$ with the isotropic angular integral $\int d \Omega=4 \pi$. Time is measured in units of the inverse of mean Fermi energy, $\bar{E}_{F}=\left(\epsilon_{F}^{\uparrow}+\epsilon_{F}^{\downarrow}\right) / 2=\bar{k}_{F}^{2} / 2 m$. The interaction strength is $\bar{k}_{F} a_{s}^{0}=0.0158$ for the quenched interaction and we take $\bar{k}_{F} a_{s}=0.0097$ for the initial interaction. 

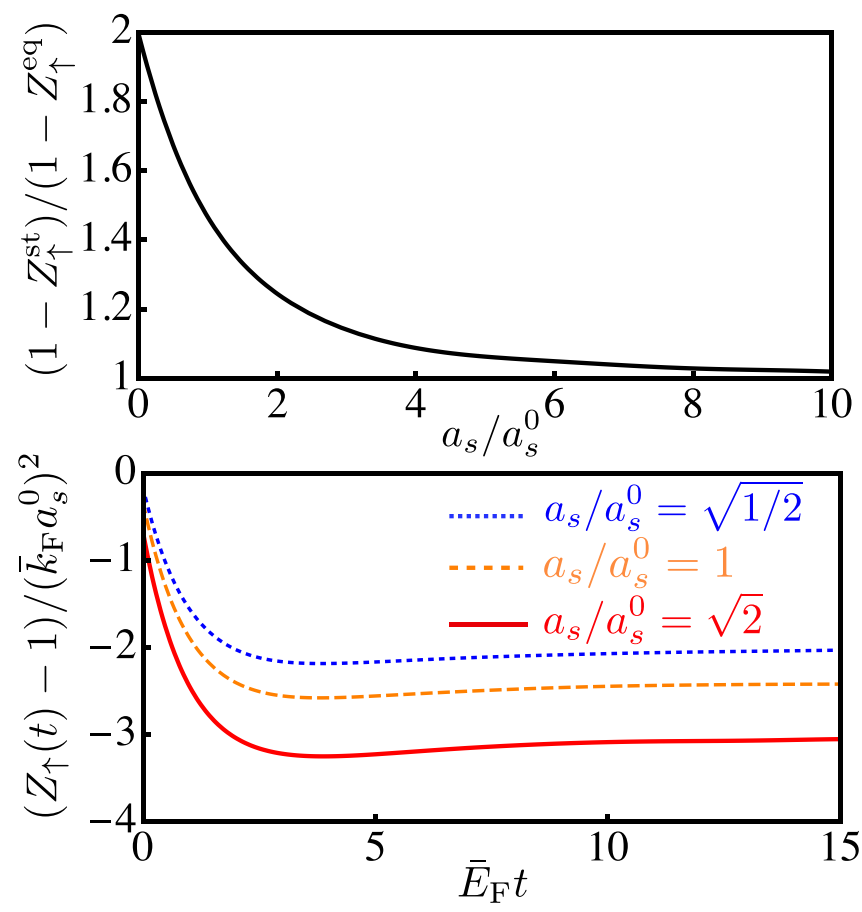

FIG. 5. Upper panel: $\left(1-Z_{\sigma}^{s t}\right) /\left(1-Z_{\sigma}^{e q}\right)$ as a function of the ratio of the scattering lengths. $Z_{\sigma}^{s t}$ and $Z_{\sigma}^{\text {eq }}$ are the stationary and equilibrium values of the discontinuity at the Fermi momentum of the momentum distribution for the spin $\sigma=\uparrow$ in a two-component Fermi gas following a sudden interaction quench. $a_{s}^{0}$ is the scattering length of the quenched interaction and $a_{s}$ is the scattering length of the initial interaction. The post-quench total scattering length is $a_{s}+a_{s}^{0}$. As discussed in the main text, the ratio $\left(1-Z_{\uparrow}^{s t}\right) /\left(1-Z_{\uparrow}^{e q}\right)$ ranges between 1 and 2 and depends on the ratio of the initial to the final interaction strengths. Lower panel: The evolution of discontinuity at Fermi momentum for different values of the ratio $a_{s} / a_{s}^{0}$ shows a plateau after a short-time transient. The interaction strength for the quenched interaction is $\bar{k}_{F} a_{s}^{0}=0.0158$. Time is measured in units of the inverse of the mean Fermi energy $\bar{E}_{F}=\left(\epsilon_{F}^{\uparrow}+\epsilon_{F}^{\downarrow}\right) / 2=\bar{k}_{F}^{2} / 2 m$. The scaling $\left(\bar{k}_{F} a_{s}^{0}\right)^{-2}$ is used because the results are obtained using second-order perturbation theory.

Eq. (24). Physically it implies that the discontinuity of the (instantaneous) momentum distribution in the prethermalized state is larger than in the ground state of the interacting system. This reflects the existence of quasiparticle excitations in the initial state (compared to the ground state and by virtue of the variational theorem). Those excitations need to be accommodated in the stationary (prethermalized) state by placing fermions in higher (and lower) momentum levels, which leads to a reduced discontinuity

\section{SUPPRESSION OF PRETHERMALIZATION}

The dynamics of the two-component gas described above illustrates that introducing a population imbalance in the initial state is not a sufficient condition to suppress prethermalization. In this section, we show that the situation changes dramatically when, besides the initial population imbalance, we allow for spin-changing collisions (SCCs) that result from the breaking of the $\mathrm{SU}(N)$ symmetry of the quenched interaction. For a contact interaction that conserves the total angular momentum, the minimum value of $N$ allowing for SCCs is $N=4$. Figure 1 schematically shows the two possible types of scattering processes. They are classified into two types: those preserving the relative spin populations (left panel) and the SCCs (right panel).

Next, we consider a sudden interaction quench in an initially interacting (with scattering length $a_{s}$ ) four-component Fermi gas. We assume the initial state contains an imbalance in the population of the different spin components as shown in Fig. 1. This population imbalance can be parametrized by the ratio $v=N_{ \pm 1 / 2} / N_{ \pm 3 / 2}$, which also determines the noninteracting state $|G S, v\rangle$ used in the perturbative treatment outlined in Sec. III. In the left panel of Fig. 6 we have plotted the time evolution of $Z_{\sigma}(t)$ for $v=1.83$. In this case, unlike the plateau observed for the imbalanced two-component gas, we find a slow decay of $Z_{\sigma}(t)$ as a function of time, and the momentum distributions show increase (decrease) for the particles above (below) the Fermi surface, which is suggestive of the absence of prethermalization. In order to shed further light into the behavior of the system, it is useful to consider the dynamics of the ratio $\delta N_{ \pm 3 / 2}(t) / N_{\text {tot }}$, where $\delta N_{\sigma}(t)$ measures the deviation from its initial value of the population for spin component $\sigma$. This ratio is shown in Fig. 6, which illustrates how the SCCs alter the relative populations by decreasing the population of the majority components with $\sigma= \pm 1 / 2$ and increasing that of the minority component [note that $\delta N_{ \pm 1 / 2}(t)=-\delta N_{ \pm 3 / 2}(t)$ because the total number is conserved].

Analytically (see Appendix B), it can be shown that, after a short transient, the rate of population change is given by Fermi's golden rule. This result can be obtained by formally taking the limit $t \rightarrow+\infty$ of the second-order expressions for $\delta N_{\sigma}(t)$, i.e.,

$$
\begin{aligned}
\lim _{t \rightarrow \infty} & \frac{\delta N_{\sigma}(t)}{t} \\
\propto & \frac{1}{V^{2}} \sum_{p k q r} \sum_{\alpha \beta \gamma} g_{f}^{(2)}(\sigma, \alpha ; \beta, \gamma)\left[n_{p \sigma}^{0} n_{k \alpha}^{0}\right. \\
& \left.\times\left(1-n_{q \beta}^{0}\right)\left(1-n_{r \gamma}^{0}\right)-\left(1-n_{p \sigma}^{0}\right)\left(1-n_{k \alpha}^{0}\right) n_{q \beta}^{0} n_{r \gamma}^{0}\right] \\
& \times \delta\left(\epsilon_{p}+\epsilon_{k}-\epsilon_{q}-\epsilon_{r}\right) \delta_{\boldsymbol{p}+\boldsymbol{k}, \boldsymbol{q}+\boldsymbol{r}},
\end{aligned}
$$

where $g_{f}^{(2)}(\sigma, \alpha ; \beta, \gamma)$ is the (square of the) matrix element for SCCs. Note that the rate of population change is independent of the initial $\mathrm{SU}(N)$-symmetric interaction $\left(U_{0}\right)$ since $g_{f}^{(2)}$ [cf. Eq. (23)] only depends on the quenched interaction $U_{1}$ through the couplings $g_{J}$ and does not depend on $U_{0}$. The slope, $d \delta N_{\sigma}(t) / d t$, in the linearly increasing regime is proportional to the phase space volume available for SCCs multiplied by the matrix element mediating the transitions. As shown in Fig. 7, the rate of change of $\delta N_{\sigma}(t)$ (that is, its slope in Fig. 7) becomes larger as the initial population imbalance increases, as expected from the corresponding enhancement in the available phase space. Since the SCCs can induce scattering processes that increase the number of particle-hole pairs, they provide a decoherence mechanism in the short-time dynamics after the quench. The momentum distribution shows no stationary behavior. Thus, for an initial state with $v \neq 1$ in the presence of SCCs, neither the relative population nor the momentum distribution for each spin component become 

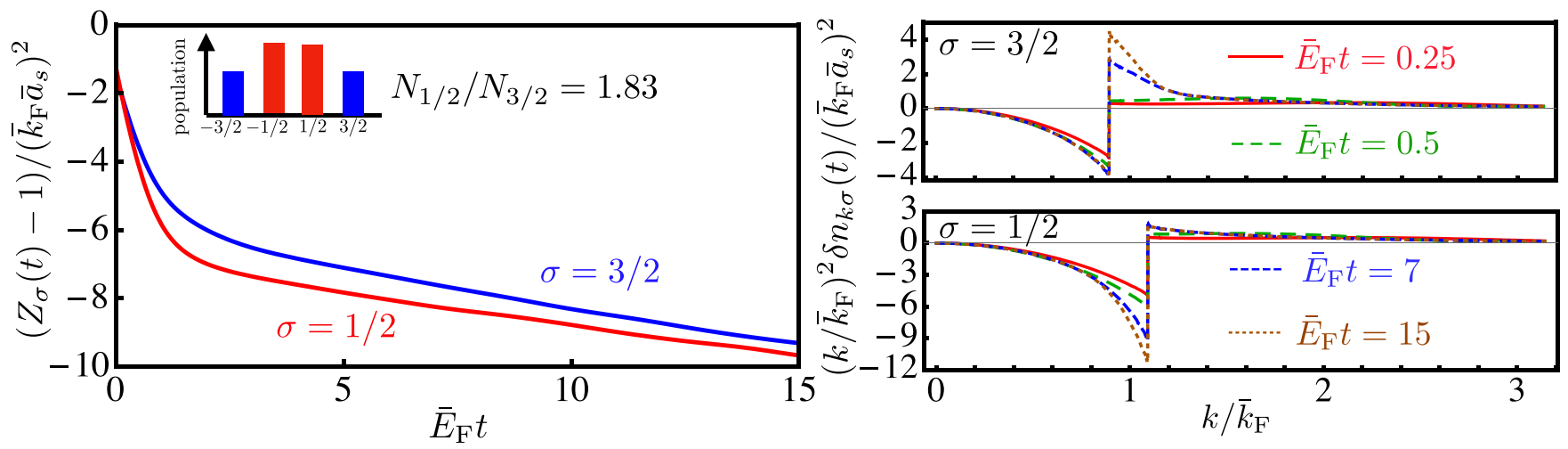

FIG. 6. Left panel: Dynamics of the discontinuity, $Z_{\sigma}(t)$, at Fermi momentum of the momentum distribution for a $N=4$ Fermi gas following a sudden quench of the interaction that breaks the $\mathrm{SU}(4)$ symmetry. The population ratio in the initial state $v=N_{ \pm 1 / 2} / N_{ \pm 3 / 2}=1.83$ is indicated in the inset. Right panel: Evolutions of the change in momentum distribution. They show an increase (decrease) in the populations of the minority (majority) component. Time is measured in units of the inverse of the mean Fermi energy $\bar{E}_{F}=\left(\epsilon_{F}^{3 / 2}+\epsilon_{F}^{1 / 2}\right) / 2=\bar{k}_{F}^{2} / 2 m$, and $\bar{a}_{s}=\left(a_{s}^{0}+a_{s}^{2}\right) / 2$ is the mean value of the scattering lengths of the quenched interaction. The interaction strengths are determined by the parameters $\bar{k}_{F} a_{s}^{0}=0.0158$ and $\bar{k}_{F} a_{s}^{2}=0.0032$, for the quenched interaction and $\bar{k}_{F} a_{s}=0.0097$ for the initial interaction. The scaling $\left(\bar{k}_{F} \bar{a}_{s}\right)^{-2}$ is used because the results are obtained from second-order perturbation theory in the scattering length of the quenched interaction.

stationary after a short-time transient, indicating that the intermediate time behavior cannot be described as prethermal. Conversely, the prethermalized regime is robust when particle-hole creating scattering processes are (Pauli) blocked at short time, which is the case of an initial state with no spin imbalance (i.e., $v=1$ ), or when the SCCs are absent because the quenched interaction is $\mathrm{SU}(N)$ symmetric.

\section{CONTROL OF PRETHERMALIZATION}

We have seen in the previous sections that prethermalization can take place provided the symmetries of the postquench Hamiltonian or the initial state are properly broken.

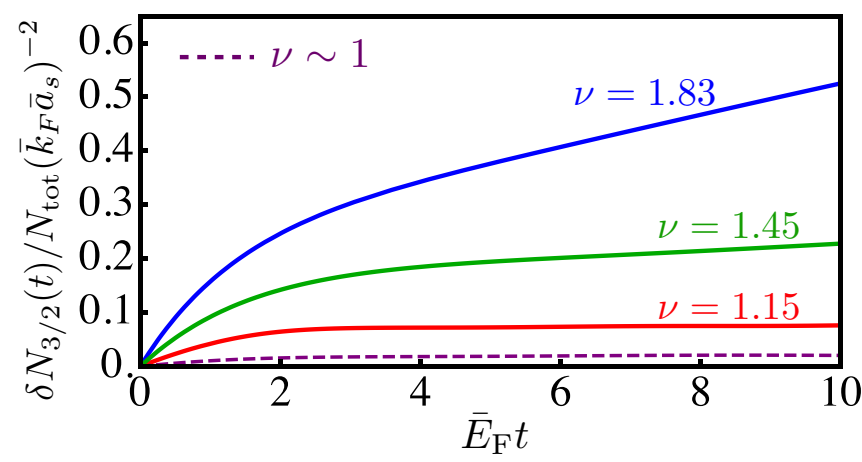

FIG. 7. Evolution of the population ratio $\delta N_{\sigma} / N_{\text {tot }}\left(\overline{k_{F}} \bar{a}_{s}\right)^{-2}$ for $\sigma=3 / 2$, and different initial conditions with symmetry $\left(N_{1 / 2}=\right.$ $\left.N_{-1 / 2}, N_{3 / 2}=N_{-3 / 2}\right)$. The scaling $\left(\bar{k}_{F} \bar{a}_{s}\right)^{-2}$ is used because the results are obtained using second-order perturbation theory in the scattering length of the quenched interaction. Time is measured in units of the inverse of the mean Fermi energy $\bar{E}_{F}=\left(\epsilon_{F}^{3 / 2}+\epsilon_{F}^{1 / 2}\right) / 2=\bar{k}_{F}^{2} / 2 m$. The interaction strengths are determined by the parameters $\bar{k}_{F} a_{s}^{0}=$ 0.0158 and $\bar{k}_{F} a_{s}^{2}=0.0032$ for the quenched interaction and $\bar{k}_{F} a_{s}=$ 0.0097 for the initial interaction. $\bar{a}_{s}=\left(a_{s}^{0}+a_{s}^{2}\right) / 2$ is the mean value of the scattering lengths of the quenched interactions.
Here we demonstrate the possibility of controlling the existence of prethermalization by carefully choosing the initial state.

As mentioned above, Eq. (27) shows that the rate of change of the relative populations vanishes when the population of the different spin species is the same and the initial state becomes $\mathrm{SU}(N=4)$ symmetric. However, this is not the only type of initial condition for which the rate of change of relative populations vanishes. Indeed, it is possible to find other types of initial states for which the rate of change of the relative population, Eq. (27), vanishes. To see this, let us define $E_{p \alpha}=$ $\epsilon_{p}-\epsilon_{F}^{\alpha}$, where $\epsilon_{F}^{\alpha}$ is the Fermi energy for the component $\alpha$. Thus, the Dirac delta function, ensuring energy conservation in Eq. (27) becomes

$$
\delta\left(\epsilon_{p}+\epsilon_{k}-\epsilon_{q}-\epsilon_{r}\right)=\delta\left(E_{p \sigma}+E_{k \alpha}-E_{q \beta}-E_{r \gamma}+\Delta_{F}\right),
$$

where $\Delta_{F}=\epsilon_{F}^{\sigma}+\epsilon_{F}^{\alpha}-\epsilon_{F}^{\beta}-\epsilon_{F}^{\gamma}$. In addition, we notice that $\delta N(t)$ receives contributions only from the SCCs, which for $N=4$ means that $\sigma=-\alpha=\frac{3}{2}$ and $\beta=-\gamma=\frac{1}{2}$. Thus,

$$
\Delta_{F}=\epsilon_{F}^{+3 / 2}+\epsilon_{F}^{-3 / 2}-\epsilon_{F}^{+1 / 2}-\epsilon_{F}^{-1 / 2} .
$$

Next we shall argue that initial states satisfying $\Delta_{F}=0$ will exhibit prethermal behavior [when initial interactions are present, the initial state must be adiabatically connected to a noninteracting state that satisfies the condition $\Delta_{F}=0$ since the initial SU $(N)$-symmetric interaction does not induce SCCs]. In order to establish this result, we first notice that the expression in Eq. (27) contains two terms. In the first one, the occupation factors $n_{p \sigma}^{0} n_{k \alpha}^{0}\left(1-n_{q \beta}^{0}\right)\left(1-n_{r \gamma}^{0}\right)$ require, at $T=0$, that is, for a pure state, that $E_{p \sigma}, E_{k \alpha} \geqslant 0$ and $E_{q \beta}, E_{r \gamma} \leqslant 0$. At the same time, the energy conservation for $\Delta_{F}=0$ requires that $E_{p \sigma}+E_{k \alpha}=E_{q \beta}+E_{r \gamma}$, which can only be satisfied if $E_{p \sigma}=E_{k \alpha}=E_{q \beta}=E_{r \gamma}=0$. The manifold of points satisfying the previous condition in the ninedimensional space span by the vectors $\boldsymbol{p}, \boldsymbol{k}, \boldsymbol{q}(\boldsymbol{r}=\boldsymbol{p}+\boldsymbol{k}-\boldsymbol{r}$ 

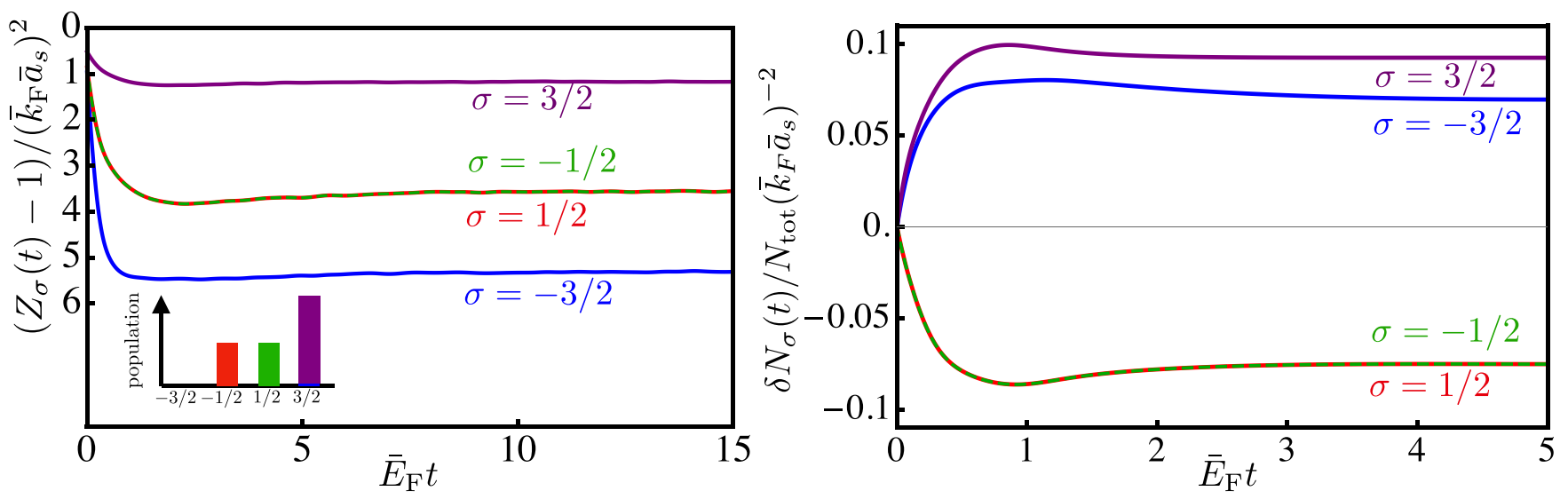

FIG. 8. Left panel: Discontinuity at the Fermi momentum starting from an imbalanced initial state. The plateau indicates the emergence of prethermalization in the presence of SCCs. The inset shows the initial condition schematically where the imbalanced initial state is $\epsilon_{F}^{-3 / 2}=0$ and $2 \epsilon_{F}^{ \pm 1 / 2}=\epsilon_{F}^{3 / 2}$. Right panel: Time evolution of the relative population. After an initial exchange of particles, the spin populations reach stationary values. Time is measured in units of the inverse of the mean Fermi energy $\bar{E}_{F}=\frac{1}{4} \sum_{\sigma} \epsilon_{F}^{\sigma}=\bar{k}_{F}^{2} / 2 m$. The interaction strengths satisfy $\bar{k}_{F} a_{s}^{0}=0.0158$ and $\bar{k}_{F} a_{s}^{2}=0.0032$ for the quenched interaction and $\bar{k}_{F} a_{s}=0.0097$ for the initial interaction. The scaling $\left(\bar{k}_{F} \bar{a}_{s}\right)^{-2}$ is for second-order perturbation calculation.

is fixed by momentum conservation) is a set of zero measure and does not contribute to the integrals over momentum in Eq. (27). An entirely identical conclusion is reached for the second term in Eq. (27). Physically, the condition that $\Delta_{F}=0$ amounts to having zero phase space for the SCCs to occur, even if this is not obvious from the fact that the initial state contains a population imbalance. This argument can be easily generalized to the case where $N>4$.

In order to explicitly show how prethermal behavior emerges when $\Delta_{F}=0$, we have computed the evolution of $Z_{\sigma}(t)$ and $\delta N_{\sigma(t)}$ by numerically evaluating the corresponding expressions for an initial state satisfying the condition that $\Delta_{F}=0$. The results are shown in Fig. 8. It is worth comparing the results on the right panel of Fig. 8, with those shown in Fig. 8 (left panel). It can be seen that, after a short transient, for the initial state satisfying the condition $\Delta_{F}=0$, the curves for $\delta N_{\sigma(t)}$ flatten out. Concurrently, $Z_{\sigma}(t)$ also reaches the characteristic prethermal plateau. However, it is worth noticing that, unlike the case of initial states with $\mathrm{SU}(N)$ symmetry which trivially satisfy $\Delta_{F}=0$, there is a change in population during the short-time transient because there are SCCs that do not satisfy energy conservation. The energy conservation is only enforced for $t$ sufficiently large. When this happens, the system enters the prethermalized regime. As a caveat, it is important to notice that if change of population happens during the short-time transient, then condition $\Delta_{F}=0$ will not ensure that the system reaches the prethermalized regime. Thus, we must require that $\delta N_{\sigma}(t) / N_{\sigma} \ll 1$, which is the case in the regime where quenched interaction can be treated perturbatively.

\section{CONCLUSIONS}

In conclusion, we have studied the prethermalization dynamics of an isolated multicomponent Fermi gas of ultracold atoms following a sudden interaction quench. This type of nonequilibrium dynamics can be experimentally studied using alkaline-earth-metal atoms, whose interaction can be tuned using optical Feshbach resonances [4,81-84], e.g., in ${ }^{173} \mathrm{Yb}$ for $N \leqslant 6$ and ${ }^{87} \mathrm{Sr}$ for $N \leqslant 10$.

We have shown that the short-time dynamics of this system is affected by both the presence of a population imbalance in the initial state and the breaking of the emergent $\mathrm{SU}(N)$ symmetry of the contact interactions by allowing spin-changing collisions. Both elements are necessary for the suppression of prethermalization, as illustrated by the behavior of the two-component Fermi gas with initial spin imbalance, which displays a robust prethermalizated regime at short to intermediate times. On the other hand, for a generic $\mathrm{SU}(N \geqslant 4)$ symmetry-breaking (i.e., imbalanced) initial state we do not observe a prethermal regime after a quenching an $\mathrm{SU}(N)$ symmetry-breaking interacting. This is because, generically, the population imbalance in the initial state provides phase space for spin-changing collisions and introduces a decoherence mechanism and suppress prethermalization.

Nevertheless, we have shown that there is a class of imbalanced initial states which allows for the emergence of prethermal behavior. This opens the possibility of using multi-component gases to study the suppression and control of this nonequilibrium state. In addition, the findings reported in this work should allow for the possibility to experimentally observe the effect of SCCs by studying the dynamics of the quantum gas following an interaction quench.

\section{ACKNOWLEDGMENTS}

M.A.C. and C.H.H. have been supported by the Ministry of Science and Technology (Taiwan) under Contracts No. NSC 102- 2112-M-007-024-MY5 and No. 107-2112-M-007-021MY5. M.A.C. also acknowledges the support of the National Center for Theoretical Sciences (NCTS) of Taiwan. Y.T. and Y.T. acknowledge support by Grants-in-Aid for Scientific Research of the Ministry of Education, Culture Sports, Science, and Technology and the Japan Society for the Promotion of Science (MEXT/JSPS KAKENHI) Grants No. 25220711, No. 17H06138, No. 18H05405, and No. 18H05228; the 

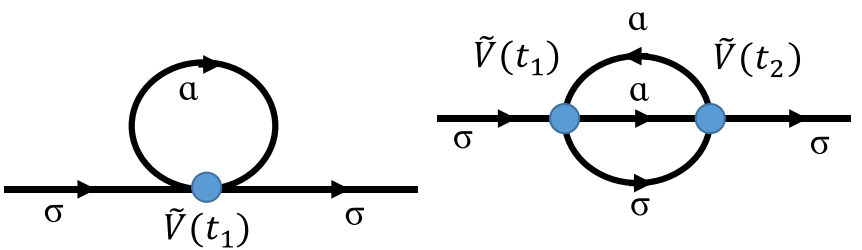

FIG. 9. First- and second-order diagram for momentum distribution. $V(t)=U_{0}(t)+U_{1}(t)$ is the sum of the total (initial plus quenched) interaction.

Impulsing Paradigm Change through Disruptive Technologies (ImPACT) program; the Japan Science and Technology Agency CREST (Grant No. JPMJCR1673); and the MEXT Quantum Leap Flagship Program (MEXT Q-LEAP, JPMXS0118069021).

\section{APPENDIX A: TECHNICAL DETAILS OF THE CALCULATIONS}

The fully connected contributions [denoted by $\langle\cdots\rangle_{c}$ in Eq. (15)] resulting from the application of Wick's theorem can be represented in terms of Feynamn graphs (cf. Fig. 9). Notice that there are four possible choices for the time arguments for the fermion propagator:

$$
i G_{0}\left(t_{1}, t_{2} ; p \sigma\right)=\left\langle\mathcal{T}\left[c_{p \sigma}\left(t_{1}\right) c_{p \sigma}^{\dagger}\left(t_{2}\right)\right]\right\rangle,
$$

where $t_{1}$ and $t_{2}$ can be in either the $\tau$ or $\bar{\tau}$ branches of $C$. The free fermion propagator can be written in matrix form as follows:

$$
\mathcal{G}_{p \sigma}(a, b)=\left(\begin{array}{ll}
i G_{p \sigma}^{T}(a, b) & i G_{p \sigma}^{<}(a, \bar{b}) \\
i G_{p \sigma}^{>}(\bar{a}, b) & i G_{p \sigma}^{\tilde{T}}(\bar{a}, \bar{b})
\end{array}\right) .
$$

Using $c_{p \sigma}(t)=c_{p \sigma} e^{-i \epsilon_{p} t}$, the entries of the above matrix can be evaluated to yield

$$
\begin{gathered}
i G_{p \sigma}^{<}\left(t_{1}, \bar{t}_{2}\right)=-n_{p \sigma}^{0} e^{i \epsilon_{p}\left(t_{2}-t_{1}\right)}, \\
i G_{p \sigma}^{>}\left(\bar{t}_{1}, t_{2}\right)=\left(1-n_{p \sigma}^{0}\right) e^{i \epsilon_{p}\left(t_{2}-t_{1}\right)}, \\
i G_{p \sigma}^{T}\left(t_{1}, t_{2}\right)=\theta\left(t_{1}-t_{2}\right) i G_{p \sigma}^{>}\left(\bar{t}_{1}, t_{2}\right)+\theta\left(t_{2}-t_{1}\right) i G_{p \sigma}^{<}\left(t_{1}, \bar{t}_{2} ;\right),
\end{gathered}
$$

$i G_{p \sigma}^{\tilde{T}}\left(\bar{t}_{1}, \bar{t}_{2}\right)=\theta\left(t_{2}-t_{1}\right) i G_{p \sigma}^{>}\left(\bar{t}_{1}, t_{2}\right)+\theta\left(t_{1}-t_{2}\right) i G_{p \sigma}^{<}\left(t_{1}, \bar{t}_{2}\right)$.

For the calculation of equal-time expectation values, we choose the time argument of the operator $(t)$ to lie slightly before the turning point of the contour $C$, which is on the timeordered $(\tau)$ branch. In this case, the fermion propagators must be obtained from Eq. (A1), which yields

$$
\mathcal{G}_{p \sigma}(t, b)=e^{-i \epsilon_{p}(t-b)}\left(\begin{array}{cc}
1-n_{p \sigma}^{0} & -n_{p \sigma}^{0} \\
0 & 0
\end{array}\right),
$$

where the nonvanishing entries correspond to $b$ lying either before or after $t$ on the contour $C$. Similarly,

$$
\mathcal{G}_{p \sigma}(a, t)=e^{-i \epsilon_{p}(a-t)}\left(\begin{array}{cc}
-n_{p \sigma}^{0} & 0 \\
1-n_{p \sigma}^{0} & 0
\end{array}\right) .
$$

and the two nonzero entries correspond to $a$ lying before or after $t$ on the contour $C$.

The self-energy can be calculated from the diagrams shown in Fig. 9 and the propagators, Eqs. (A3) to (A6). Thus, to first order in $V(t)$, we obtain

$$
\begin{gathered}
\Sigma_{\sigma}^{(0,1)}\left(t_{1}\right)=\frac{\theta\left(t_{1}\right)}{V} \sum_{\alpha} g_{f}^{(1)}(\sigma, \alpha ; \sigma, \alpha) \sum_{\boldsymbol{k}} n_{k \alpha}^{0}, \\
\Sigma_{\sigma}^{(1,0)}\left(t_{1}\right)=\frac{g_{i}}{V} \sum_{\alpha \neq \sigma} \sum_{k} n_{k \alpha}^{0} e^{-\eta\left|t_{1}\right|},
\end{gathered}
$$

where we have introduced

$$
g_{f}^{(1)}(\sigma, \alpha ; \sigma, \alpha)=\sum_{J=0,2, \ldots} \sum_{M} g_{J}\langle J M \mid F F \sigma \alpha\rangle\langle F F \sigma \alpha \mid J M\rangle .
$$

Combining the expression in matrix form, Eq. (17), the propagators, Eqs. (A7) and (A8), and the self-energy for the first-order correction, Eqs. (A9) and (A10), we obtain that firstorder correction to the instantaneous momentum distribution vanishes, i.e., $n_{p \sigma}^{(1)}=0$.

At second order in the quenched interaction, we need to use the following self-energy matrix, which contains four different combinations of the time arguments $\left(t_{1}, t_{2}\right)$ on the two branches of the closed contour:

$$
\begin{aligned}
\Sigma_{p \sigma}^{(2,0)}(b, a)= & -\frac{2 g_{i}^{2}}{V^{2}} e^{-\eta(|b|+|a|)} \sum_{k q r} \delta_{p+k, q+r} \\
& \times \sum_{\alpha \beta \gamma} \overline{\boldsymbol{\Sigma}}^{(\mathbf{2})}(b, a) \delta_{\sigma, \beta} \delta_{\alpha \gamma} \\
\Sigma_{p \sigma}^{(1,1)}(b, a)= & -\frac{4}{V^{2}} \theta(a) e^{-\eta|b|} \sum_{k q r} \delta_{p+k, q+r} \\
& \times \sum_{\alpha \beta \gamma} g_{i} g_{f}^{(1)}(\sigma, \alpha ; \sigma, \alpha) \overline{\boldsymbol{\Sigma}}^{(\mathbf{2})}(b, a) \delta_{\sigma, \beta} \delta_{\alpha, \gamma}, \\
\Sigma_{p \sigma}^{(0,2)}(b, a)= & -\frac{2}{V^{2}} \theta(b) \theta(a) \sum_{k q r} \delta_{p+k, q+r} \\
& \times \sum_{\alpha \beta \gamma} g_{f}^{(2)}(\sigma, \alpha ; \beta, \gamma) \overline{\mathbf{\Sigma}}^{(\mathbf{2})}(b, a),
\end{aligned}
$$

where $\overline{\boldsymbol{\Sigma}}^{(\mathbf{2})}(b, a)$ is the following matrix:

$$
\overline{\boldsymbol{\Sigma}}(b, a)=\left(\begin{array}{ll}
\bar{\Sigma}^{(2, T)}(b, a) & \bar{\Sigma}^{(2,>)}(\bar{b}, a) \\
\bar{\Sigma}^{(2,<)}(b, \bar{a}) & \bar{\Sigma}^{(2, \tilde{T})}(\bar{b}, \bar{a})
\end{array}\right)
$$

where, for the sake of brevity, we have suppressed the explicit dependence of $\Sigma^{(2)}(b, a)=\Sigma_{k q r, \alpha \beta \gamma}^{(2)}(b, a)$ on the momentum and spin indices. Furthermore, we have introduced the following notation:

$$
\begin{aligned}
g_{f}^{(2)}(\sigma, \alpha ; \beta, \gamma) & \\
= & \sum_{J_{1}, J_{2}=0,2, \ldots} \sum_{M_{1} M_{2}} g_{J_{1}} g_{J_{2}}\left\langle J_{1} M_{1} \mid F F \sigma \alpha\right\rangle \\
& \times\left\langle F F \beta \gamma \mid J_{1} M_{1}\right\rangle\left\langle J_{2} M_{2} \mid F F \sigma \alpha\right\rangle\left\langle F F \beta \gamma \mid J_{2} M_{2}\right\rangle,
\end{aligned}
$$


which contains the information of coupling strength and Clebsch-Gordon coefficients. Using the diagram in Fig. 9 and free propagators in Eqs. (A3)-(A8), we can write down the following expression for the elements in the matrix:

$$
\begin{aligned}
& \bar{\Sigma}^{(2,<)}\left(t_{2}, \bar{t}_{1}\right)=i^{3} G_{k \alpha}^{<}\left(t_{2}, \bar{t}_{1}\right) G_{q \beta}^{>}\left(\bar{t}_{1}, t_{2}\right) G_{r \gamma}^{>}\left(\bar{t}_{1}, t_{2}\right), \\
& =\left(1-n_{q \beta}^{0}\right)\left(1-n_{r \gamma}^{0}\right) n_{k \alpha}^{0} e^{i\left(t_{1}-t_{2}\right)\left(\epsilon_{q}+\epsilon_{r}-\epsilon_{k}\right)}, \\
& \bar{\Sigma}^{(2,>)}\left(\bar{t}_{2}, t_{1}\right)=i^{3} G_{k \alpha}^{>}\left(\bar{t}_{2}, t_{1}\right) G_{q \beta}^{<}\left(t_{1}, \bar{t}_{2}\right) G_{r \gamma}^{<}\left(t_{1}, \bar{t}_{2}\right), \\
& =n_{q \beta}^{0} n_{r \gamma}^{0}\left(1-n_{k \alpha}^{0}\right) e^{i\left(t_{1}-t_{2}\right)\left(\epsilon_{q}+\epsilon_{r}-\epsilon_{k}\right)}, \\
& =\theta\left(t_{2}-t_{1}\right) \Sigma^{(2,>)}\left(t_{2}, \bar{t}_{1}\right)+\theta\left(t_{1}-t_{2}\right) \Sigma^{(2,>)}\left(\bar{t}_{2}, t_{1}\right), \\
& \bar{\Sigma}^{(2, \tilde{T})}\left(\bar{t}_{2}, \bar{t}_{1}\right) \\
& =i^{3} G_{k \alpha}^{\bar{T}}\left(\bar{t}_{2}, \bar{t}_{1}\right) G_{q \beta}^{\bar{T}}\left(\bar{t}_{1}, \bar{t}_{2}\right) G_{r \gamma}^{\bar{T}}\left(\bar{t}_{1}, \bar{t}_{2}\right), \\
& =\theta\left(t_{1}-t_{2}\right) \Sigma^{(2,>)}\left(t_{2}, \bar{t}_{1}\right)+\theta\left(t_{2}-t_{1}\right) \Sigma^{(2,>)}\left(\bar{t}_{2}, t_{1}\right) \text {. }
\end{aligned}
$$

\section{APPENDIX B: EVOLUTION OF THE SPIN POPULATION}

Using the result of momentum distribution, we can find the change in populations to leading order:

$$
\begin{aligned}
\delta N_{\sigma} & =\sum_{p} n_{p \sigma}^{(2)}(t) \\
& =-\frac{2}{V^{2}} \sum_{p k q r} \sum_{\sigma \alpha \beta \gamma} Q_{p k q r}^{\sigma \alpha \beta \gamma} g^{(2)}(\sigma, \alpha ; \beta, \gamma) F\left(E_{p k q r}, t\right) .
\end{aligned}
$$

Notice that this result does not involve the corrections of $O\left(U_{0}^{2}\right)$ and $O\left(U_{1} U_{0}\right)$ to the momentum distribution. This is because the initial interaction conserves the population of the different spin components.

For intermediate to long times, we notice that, formally,

$$
\lim _{t \rightarrow+\infty} F(E, t) \propto t \delta(E)
$$

and therefore the rate of population change of the different components is given by the golden-rule expression in Eq. (27). This rate is proportional to the phase-space volume available for scattering with SCCs. We note that this is result is independent of the initial interaction which preserves the $\mathrm{SU}(N)$ symmetry.
[1] M. A. Cazalilla, A. F. Ho, and M. Ueda, New J. Phys. 11, 103033 (2009).

[2] A. V. Gorshkov, M. Hermele, V. Gurarie, C. Xu, P. S. Julienne, J. Ye, P. Zoller, E. Demler, M. D. Lukin, and A. M. Rey, Nat. Phys. 6, 289 (2010).

[3] M. A. Cazalilla and A. M. Rey, Rep. Prog. Phys. 77, 124401 (2014).

[4] R. Yamazaki, S. Taie, S. Sugawa, and Y. Takahashi, Phys. Rev. Lett. 105, 050405 (2010).

[5] S. Stellmer, R. Grimm, and F. Schreck, Phys. Rev. A 84, 043611 (2011).

[6] I. Affleck and J. B. Marston, Phys. Rev. B 37, 3774 (1988).

[7] J. B. Marston and I. Affleck, Phys. Rev. B 39, 11538 (1989).

[8] P. Coleman, Phys. Rev. B 28, 5255 (1983).

[9] N. Read and D. M. Newns, J. Phys. C 16, L1055 (1983).

[10] N. Read and D. Newns, Adv. Phys. 36, L1055 (1987).

[11] N. Read and S. Sachdev, Nucl. Phys. B 316, 609 (1989).

[12] C.-H. Cheng and S.-K. Yip, Phys. Rev. A 95, 033619 (2017).

[13] S. Barbarino, L. Taddia, D. Rossini, L. Mazza, and R. Fazio, Nat. Commun. 6, 8134 (2015).

[14] S. Barbarino, L. Taddia, D. Rossini, L. Mazza, and R. Fazio, New J. Phys. 18, 035010 (2016).

[15] A. Celi, P. Massignan, J. Ruseckas, N. Goldman, I. B. Spielman, G. Juzeliunas, and M. Lewenstein, Phys. Rev. Lett. 112, 043001 (2014).

[16] M. E. Beverland, G. Alagic, M. J. Martin, A. P. Koller, A. M. Rey, and A. V. Gorshkov, Phys. Rev. A 93, 051601(R) (2016).

[17] M. Hermele, V. Gurarie, and A. M. Rey, Phys. Rev. Lett. 103, 135301 (2009).

[18] J. Dufour and F. Mila, Phys. Rev. A 94, 033617 (2016).

[19] P. Nataf and F. Mila, Phys. Rev. B 93, 155134 (2016).

[20] S. Sugawa, K. Inaba, S. Taie, R. Yamazaki, M. Yamashita, and Y. Takahashi, Nat. Phys. 7, 642 (2011).
[21] S. Taie, R. Yamazaki, S. Sugawa, and Y. Takahashi, Nat. Phys. 8, 825 (2012).

[22] H. Ozawa, S. Taie, Y. Takasu, and Y. Takahashi, Phys. Rev. Lett. 121, 225303 (2018).

[23] C. Hofrichter, L. Riegger, F. Scazza, M. Höfer, D. R. Fernandes, I. Bloch, and S. Fölling, Phys. Rev. X 6, 021030 (2016).

[24] X. Zhang, M. Bishof, S. L. Bromley, C. V. Kraus, M. S. Safronova, P. Zoller, A. M. Rey, and J. Ye, Science 345, 1467 (2014).

[25] G. Cappellini, M. Mancini, G. Pagano, P. Lombardi, L. Livi, M. Siciliani de Cumis, P. Cancio, M. Pizzocaro, D. Calonico, F. Levi, C. Sias, J. Catani, M. Inguscio, and L. Fallani, Phys. Rev. Lett. 113, 120402 (2014).

[26] F. Scazza, C. Hofrichter, M. Hofer, P. C. De Groot, I. Bloch, and S. Folling, Nat. Phys. 10, 779 (2014).

[27] T. Ozawa and G. Baym, Phys. Rev. A 82, 063615 (2010).

[28] D. Banerjee, M. Bögli, M. Dalmonte, E. Rico, P. Stebler, U.-J. Wiese, and P. Zoller, Phys. Rev. Lett. 110, 125303 (2013).

[29] J. Berges, S. Borsányi, and C. Wetterich, Phys. Rev. Lett. 93, 142002 (2004).

[30] M. Moeckel and S. Kehrein, Phys. Rev. Lett. 100, 175702 (2008).

[31] M. Moeckel and S. Kehrein, Ann. Phys. (NY) 324, 2146 (2009).

[32] N. Nessi, A. Iucci, and M. A. Cazalilla, Phys. Rev. Lett. 113, 210402 (2014)

[33] S. A. Hamerla and G. S. Uhrig, Phys. Rev. B 89, 104301 (2014).

[34] M. Eckstein, M. Kollar, and P. Werner, Phys. Rev. Lett. 103, 056403 (2009).

[35] N. Nessi and A. Iucci, arXiv:1503.02507.

[36] M. Marcuzzi, J. Marino, A. Gambassi, and A. Silva, Phys. Rev. Lett. 111, 197203 (2013).

[37] F. H. L. Essler, S. Kehrein, S. R. Manmana, and N. J. Robinson, Phys. Rev. B 89, 165104 (2014). 
[38] T. Langen, T. Gasenzer, and J. Schmiedmayer, J. Stat. Mech. (2016) 064009.

[39] M. Moeckel and S. Kehrein, New J. Phys. 12, 055016 (2010).

[40] L. Rademaker, SciPost Phys. 7, 071 (2019).

[41] A. Mitra, Phys. Rev. B 87, 205109 (2013).

[42] M. A. Cazalilla, Phys. Rev. Lett. 97, 156403 (2006).

[43] M. Buchhold, M. Heyl, and S. Diehl, Phys. Rev. A 94, 013601 (2016).

[44] Z.-X. Gong and L.-M. Duan, New J. Phys. 15, 113051 (2013).

[45] M. Kollar, F. A. Wolf, and M. Eckstein, Phys. Rev. B 84, 054304 (2011).

[46] M. A. Cazalilla and M.-C. Chung, J. Stat. Mech. (2016) 064004.

[47] M. Babadi, E. Demler, and M. Knap, Phys. Rev. X 5, 041005 (2015).

[48] V. Alba and M. Fagotti, Phys. Rev. Lett. 119, 010601 (2017).

[49] P. Zou and Z.-M. Zhang, J. Phys. B 51, 145003 (2018).

[50] L. Vidmarand M. Rigol, J. Stat. Mech. (2016) 064007.

[51] M. Rigol, V. Dunjko, V. Yurovsky, and M. Olshanii, Phys. Rev. Lett. 98, 050405 (2007).

[52] P. Calabrese, F. H. L. Essler, and M. Fagotti, Phys. Rev. Lett. 106, 227203 (2011).

[53] E. Ilievski, J. De Nardis, B. Wouters, J.-S. Caux, F. H. L. Essler, and T. Prosen, Phys. Rev. Lett. 115, 157201 (2015).

[54] T. M. Wright, M. Rigol, M. J. Davis, and K. V. Kheruntsyan, Phys. Rev. Lett. 113, 050601 (2014).

[55] N. Andrei, arXiv:1606.08911.

[56] M. Kormos, M. Collura, and P. Calabrese, Phys. Rev. A 89, 013609 (2014).

[57] M. Kormos, A. Shashi, Y.-Z. Chou, J.-S. Caux, and A. Imambekov, Phys. Rev. B 88, 205131 (2013).

[58] T. Langen, S. Erne, R. Geiger, B. Rauer, T. Schweigler, M. Kuhnert, W. Rohringer, I. E. Mazets, T. Gasenzer, and J. Schmiedmayer, Science 348, 207 (2015).

[59] M. Rigol, V. Dunjko, and M. Olshanii, Nature (London) 452, 854 (2008).

[60] L. F. Santos and M. Rigol, Phys. Rev. E 82, 031130 (2010).

[61] L. F. Santos and M. Rigol, Phys. Rev. E 81, 036206 (2010).

[62] M. Rigol, Phys. Rev. A 80, 053607 (2009).

[63] M. Rigol, Phys. Rev. Lett. 103, 100403 (2009).

[64] G. Biroli, C. Kollath, and A. M. Läuchli, Phys. Rev. Lett. 105, 250401 (2010).

[65] T. Mori, T. N. Ikeda, E. Kaminishi, and M. Ueda, J. Phys. B 51, 112001 (2018).
[66] K. Mallayya and M. Rigol, Phys. Rev. Lett. 120, 070603 (2018).

[67] Y. Tang, W. Kao, K.-Y. Li, S. Seo, K. Mallayya, M. Rigol, S. Gopalakrishnan, and B. L. Lev, Phys. Rev. X 8, 021030 (2018).

[68] T. Langen, T. Schweigler, E. Demler, and J. Schmiedmayer, New J. Phys. 20, 023034 (2018).

[69] M. Eckstein, M. Kollar, and P. Werner, Phys. Rev. B 81, 115131 (2010).

[70] M. Van Regemortel, H. Kurkjian, I. Carusotto, and M. Wouters, Phys. Rev. A 98, 053612 (2018).

[71] R. Barnett, A. Polkovnikov, and M. Vengalattore, Phys. Rev. A 84, 023606 (2011).

[72] J. G. Cosme, Phys. Rev. A 97, 043610 (2018).

[73] B. Neyenhuis, J. Smith, A. C. Lee, J. Zhang, P. Richerme, P. W. Hess, Z.-X. Gong, A. V. Gorshkov, and C. Monroe, Sci. Adv. 3, e1700672 (2017).

[74] M. Gring, M. Kuhnert, T. Langen, T. Kitagawa, B. Rauer, M. Schreitl, I. Mazets, D. A. Smith, E. Demler, and J. Schmiedmayer, Science 337, 1318 (2012).

[75] C. Eigen, J. A. P. Glidden, R. Lopes, E. A. Cornell, R. P. Smith, and Z. Hadzibabic, Nature (London) 563, 221 (2018).

[76] M. Stark and M. Kollar, arXiv:1308.1610.

[77] A. Chiocchetta, M. Tavora, A. Gambassi, and A. Mitra, Phys. Rev. B 94, 134311 (2016).

[78] S. Huber, M. Buchhold, J. Schmiedmayer, and S. Diehl, Phys. Rev. A 97, 043611 (2018).

[79] C.-H. Huang and M. A. Cazalilla, Phys. Rev. A 99, 063612 (2019).

[80] S.-K. Yip and T.-L. Ho, Phys. Rev. A 59, 4653 (1999).

[81] S. Blatt, T. L. Nicholson, B. J. Bloom, J. R. Williams, J. W. Thomsen, P. S. Julienne, and J. Ye, Phys. Rev. Lett. 107, 073202 (2011).

[82] R. Ciuryło, E. Tiesinga, and P. S. Julienne, Phys. Rev. A 71, 030701(R) (2005).

[83] K. Enomoto, K. Kasa, M. Kitagawa, and Y. Takahashi, Phys. Rev. Lett. 101, 203201 (2008).

[84] M. Yan, B. J. DeSalvo, B. Ramachandhran, H. Pu, and T. C. Killian, Phys. Rev. Lett. 110, 123201 (2013).

[85] P. O. Fedichev, Y. Kagan, G. V. Shlyapnikov, and J. T. M. Walraven, Phys. Rev. Lett. 77, 2913 (1996).

[86] M. Theis, G. Thalhammer, K. Winkler, M. Hellwig, G. Ruff, R. Grimm, and J. H. Denschlag, Phys. Rev. Lett. 93, 123001 (2004).

[87] C. Gramsch and M. Potthoff, Phys. Rev. B 92, 235135 (2015).

[88] N. Tsuji and P. Werner, Phys. Rev. B 88, 165115 (2013). 